\section{HIGHLIGHTS}

- High temperature superconducting (HTS) bulks offer the potential of trapping and maintaining much higher magnetic loading level compared with the conventional permanent magnets used in rotary machines, although the effective magnetization of multiple HTS bulks with different relative orientations over the surface of cylindrical rotors creates new challenges. In this paper, we present the design and numerical validation of the Pulse Field Magnetization (PFM) strategy considered for the magnetization of the four-pole synchronous fully superconducting motor developed at the University of Cambridge. Different compositions of the magnetic poles have been obtained depending on the relative orientation of the magnetizing coil and the surfaces of the columns of bulks that conform a magnetic pole. For the first PFM strategy, the experimental measurements have shown that only six out of fifteen columns of five HTS bulks each, have been magnetized along the radial direction. Thus, in order to explain these observations, two bidimensional models accounting for the electromagnetic response of the top and lateral cross sections of three columns of HTS bulks subjected to multiple pulsed magnetic fields, and after the thermal relaxation of the entire system, have been created. Both models are built in COMSOL Multiphysics, based upon the so-called H-Formulation for the solution of the Maxwell equations over the superconducting domains, and the Fourier's heat transfer equation for accounting the typical pool boiling characteristics of the liquid nitrogen over their surfaces. Our numerical results have shown an excellent agreement with the experimental observations, revealing the occurrence of a nearly orthogonal distribution of current profiles in the outer columns of a three column pole, this regarding the main direction of the magnetization in the central column which is directly subjected to the PFM. Then, in order to maximize the magnetized area of the four-pole superconducting rotor taking advantage of the whole number of HTS bulks, an extended PFM strategy has been proposed by considering the magnetization of at least three successive columns of HTS bulks per pole. In the extended PFM strategy although the area of each one of the poles can be seen increased by a factor of $200 \%-400 \%$, the maximum peaks of trapped magnetic field over the cylindrical distribution of HTS bulks can be reduced in up to a $50 \%$ of their original radial magnetization. Thus, although the effective area of the poles can be enlarged by using a significantly larger number of magnetized HTS bulks, the amount of trapped magnetic field is lower and the synchronization of the rotation of the shaft with the frequency of the supply current can be significantly challenging when there is an uneven distribution of HTS bulks over the active surface of the rotor, or when there are even slight differences between the sizes of the HTS bulks and their physical properties. 


\title{
Pulsed Field Magnetization Strategies and the Field Poles Composition in a Bulk-type Superconducting Motor
}

\author{
Zhen Huang, ${ }^{1, *}$ H. S. Ruiz, ${ }^{2}$ and T. A. Coombs ${ }^{3}$ \\ ${ }^{1}$ Academy of Information Technology and Electrical Engineering, \\ Shanghai Jiao Tong University, 800 Dongchuan Road, Shanghai 200240, China \\ ${ }^{2}$ Department of Engineering, University of Leicester, \\ University Road, Leicester LE1 7RH, United Kingdom ${ }^{\dagger}$ \\ ${ }^{3}$ Department of Engineering, University of Cambridge, \\ 9 JJ Thomson Avenue, Cambridge CB3 OFA, United Kingdom
}

(Dated: January 23, 2017)

\begin{abstract}
High temperature superconducting (HTS) bulks offer the potential of trapping and maintaining much higher magnetic loading level compared with the conventional permanent magnets used in rotary machines, although the effective magnetization of multiple HTS bulks with different relative orientations over the surface of cylindrical rotors creates new challenges. In this paper, we present the design and numerical validation of the Pulse Field Magnetization (PFM) strategy considered for the magnetization of the four-pole synchronous fully superconducting motor developed at the University of Cambridge. In a first instance, singular columns of up to five HTS bulks aligned over the height of the rotor were subjected to up to three magnetic pulses of $1.5 \mathrm{~T}$ peak, and the experimental results have been simulated by considering the electrical and thermal properties of the system in a 2D approach. The entire active surface of the rotor is covered by HTS bulks of approximately the same dimensions, resulting in an uneven distribution of pole areas with at least one of the poles formed by up to 3 columns of magnetized bulks, with relatively the same peaks of trapped magnetic field. Thus, in order to effectively use the entire area of the superconducting rotor, multiple pulsed fields per column have been applied under the same experimental conditions, what results in about three times larger magnetic pole areas but with an average drop on the peaks of trapped magnetic field of about $50 \%$.
\end{abstract}

PACS numbers: 74.25.Vs, 74.25.Ha, 41.20.Z, 02.30.Xx

\section{INTRODUCTION}

The first conceptual design of a fully high temperature superconducting (HTS) motor was proposed at the University of Cambridge in 2006 [1]. In the original conceptual design, the four-pole rotor of the motor had a nonmagnetic field core and an HTS field winding with six-hundred turns made of the second generation $(2 \mathrm{G})$ of YBCO-coated tape. Likewise, the stator was conceived through the arrangement of six racetrack coils for the air gap HTS armature windings, and an iron shield for screening the environment from stray magnetic fields. However, the estimated cost for the use of 2G HTS tape on field windings above 1 Tesla, led in 2008 to a full redesigning of the rotor by) substitution of the field windings with high quality YBCO bulks [2]. The final prototype of the motor operating at LN2 bath was finally announced in 2014 [3]. This fully HTS motor has been controlled to start up at various speed by a variable voltage variable frequency open-loop control system [4], and the HTS motor has been tested for continuous operation periods longer than 5 hours, with an overall efficiency of $78.8 \%$ at $5 \mathrm{~Hz}$. However, due to the use of an inhomogeneous distribution of HTS bulks, it aimed to cover the

\footnotetext{
* Electronic address: zhen.huang@sjtu.edu.cn

$\dagger$ Electronic address: dr.harold.ruiz@le.ac.uk

$\ddagger$ Electronic address: tac1000@cam.ac.uk
}

entire surface of the rotor core (Fig. 1) as well as, the impossibility to fabricate each one of the four poles of the rotor as a single piece of $\mathrm{YBCO}$, an inhomogeneous distribution of trapped field has been observed under different magnetising conditions [5]. This phenomenon has been observed in simplified scenarios where the motor makes an effective use of only one column of HTS bulks for each magnetic pole. Thus, in this paper we present an extended strategy for the Pulsed Field Magnetisation (PFM) of the rotor poles, each conformed of up to three columns of five HTS bulks, i.e., with a total of fifteen HTS bulks per pole as it is shown in the inset of Fig. 1. It is worth mentioning that in the past most of the theoretical and experimental research on PFM for rotary machines has been focused on the use of a single bulk per pole [6-10], which makes of this study a step forward on the understanding of the optimal use of HTS bulks on the rotor of novel superconducting motors.

This paper is organised as follows: In Section II, we present the experimental results and numerical modelling of what onwards is called the simplified PFM approach, i.e., after considering the magnetization of rotor poles made of one single column of up to five HTS bulks, each of $33 \mathrm{~mm}$ diameter and $5 \mathrm{~mm}$ thickness. Measurements are presented for all over the surface of the HTS rotor $(8.5 \mathrm{~cm}$ radius, $22 \mathrm{~cm}$ height) within an angular average accuracy of $\pm 1^{\circ}$. Then, in Section III an extended PFM strategy is implemented by considering the magnetisation of the whole set of bulks covering the entire active area 


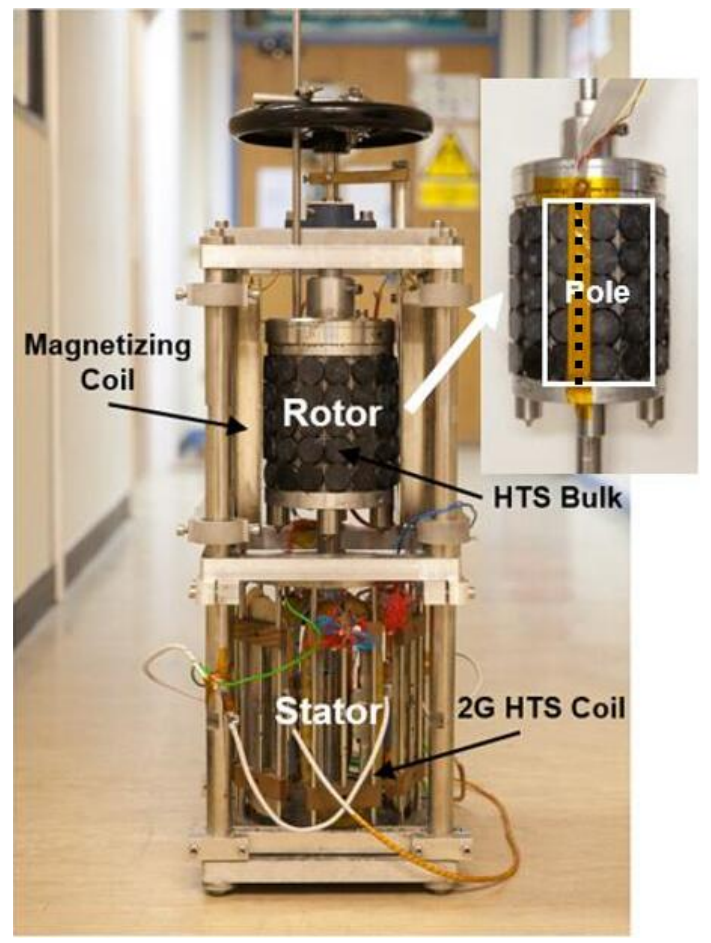

Figure 1. (Colour online) Prototype of the fully HTS motor developed at the University of Cambridge. The insert shows the superconducting rotor which has fifteen columns of YBCO bulks covering the entire surface of the rotor core of $8.5 \mathrm{~cm}$ radius and $22 \mathrm{~cm}$ height.

of the rotor, leading to an increase of about 3 times of effective magnetised area per pole. Finally, in Section IV the main conclusions of this study are summarized.

\section{SIMPLIFIED PFM STRATEGY AND NUMERICAL MODELLING}

For the sake of discussion and brevity of this manuscript, the reader is referred to Ref. [5] where the structural and electrical design parameters of the fully HTS motor and the technical aspects of the PFM experimental setup developed at the facilities of the University of Cambridge, have been reported. Here, it worth just mentioning that for the so-called simplified PFM strategy, a magnetic pole is created by aligning one of the columns of HTS bulks with the wider face of the magnetising coil displayed in Fig. 1. Then, with the mean position of the column of HTS bulks firmly locked with the line of maximum external magnetic field, three magnetic pulses within intervals of 15 min each, for assuring thermal and magnetic relaxation, are applied.

This is a four-pole machine, six pulses in total are given to make the rotor become a four-pole rotor, which means that the first three pulses are given to make a pair of $\mathrm{N}$
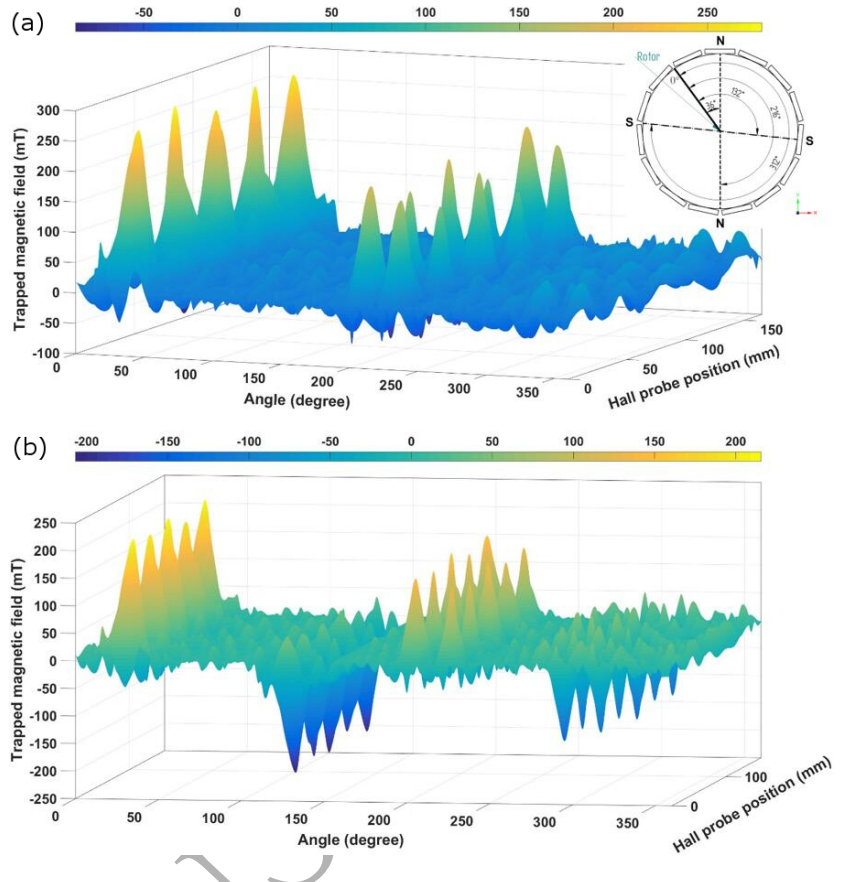

Figure 2. (Colour online) (a) $360^{\circ}$ field profile $\left(B_{r}\right)$ measured $4 \mathrm{~mm}$ away from the rotor surface after three pulses to produce a pair of $\mathrm{N}$ poles, and (b) after subsequent three pulses to produce a pair of $\mathrm{S}$ poles. The black box at the top indicates the position of the first N-pole, and in the bottom both the first N- and S- poles are highlighted. Each of these poles contains three columns of bulks with the $y$-axis along the height of the rotor, i,.e., referring to the position of the set of 11 hall probes shown in the inset of Fig. 1. The angular position of the resulting four-poles is illustrated in accordance with the rotor design as it is shown in the top-right inset.

poles and the next three pulses are given to make a pair of $\mathrm{S}$ poles. The position of pulses is indicated in Fig. 3 . According to Fig. 3(a)'s configuration, one magnetizing coil is located at the center of one column bulks (single column magnetization) and the other magnetizing coil is located in the middle of two columns bulks (two columns magnetization). These three columns are magnetized at the same time for composing one single pair of poles. In Fig. 2, the $360^{\circ}$ magnetic field profile $\left(B_{r}\right)$ after the PFM of the pair of $\mathrm{N}$ poles (Fig. 2(a)) and the subsequent magnetization of the S poles (Fig. 2(b)), measured $4 \mathrm{~mm}$ away from the surface of the rotor and along the height of the columns of HTS bulks, is shown. These experimental measurements are within an angular experimental resolution of $\pm 1^{\circ}$, starting from the black solid line referred as $0^{\circ}$, which means $36^{\circ}$ behind the centre of the first $\mathrm{N}$ pole (see inset in Fig. 2). It is to be noticed that under this configuration each one of the rotor poles can be composed by a maximum of three columns of five HTS bulks, but only the central column of the first N-pole and the first Spole is affected by the PFM. This strategy creates a total magnet area of approximately $50.5 \mathrm{~cm}^{2}$ per each one of 
these poles ( $31 \mathrm{~mm}$ width $\times 163 \mathrm{~mm}$ height) with slightly different magnetic peaks for each of the composing five HTS bulks. It is due to the inherent inhomogeneities of the samples and the consequences of the mechanical and thermal stresses that the bulks have suffered after many experimental tests. The maximum trapped magnetic field by each one of the five bulks constituting the first $\mathrm{N}$-pole (at $36^{\circ}$ ) is $228 \mathrm{mT}, 241 \mathrm{mT}, 220 \mathrm{mT}, 238 \mathrm{mT}$, and $235 \mathrm{mT}$, respectively.

The other $\mathrm{N}$ pole is located at $216^{\circ}$ exposing a different magnetisation profile, it compared to the magnetization profile observed for the first N-pole as shown in Fig. 2 (a). This phenomenon is a consequence of the odd number of columns of HTS bulks which have been used, it in order to cover the entire surface of the rotor. However, it has allowed us to explore the possibility to magnetise two columns of bulks simultaneously, i.e., when the pulse field is centred in between of two columns of bulks rather than the central cross line of a single column. As it can be observed in Fig. 2 (b), for the PFM on the second pair of poles the applied magnetic field is not fully perpendicular to the wider surface of any of the HTS bulks, what reduces the area of the loops of magnetisation currents and therefore the amount of trapped magnetic field. Then, the average trapped magnetic field of the two columns conforming the second N-pole results lower than the maximum magnetic field trapped by the single column of HTS bulks in the first N-pole. In fact, in the case of the second N-pole, it is possible to observé how two columns of bulks have been magnetised with an average peak magnetic field $38 \%$ lower than the average peak field observed at $36^{\circ}$, i.e, at the single column of the first N-pole $(232.4 \mathrm{mT})$. However, for each one of the second pair of poles, a total of ten bulks have been effectively magnetised what duplicates the rotor pole area. It is also possible to observe that the set of three magnetic pulses that have been applied to the second pair of poles (pointing downwards in Fig. 2 (b)) do not affect the amount of trapped magnetic field by the first $\mathrm{N}$ and S-pole (pointing upwards in Fig. 2(a)). Finally, it is worth mentioning that although the magnetised area for the four poles rotor is uneven, it has been already proven that it does not represent a major issue for the operation of the motor thanks to the embedded speed control algorithm [4].

Then, in order to unveil the resulting distribution of magnetic field along the height and arc width of the magnetic poles, as well as the current distribution inside of the HTS bulks, a couple of two-dimensional numerical models have been created by simplifying the PFM system in terms of the two different view angles depicted in Fig. 3. In agreement with the experimental results for the first pair of $\mathrm{N}$ - and S- poles presented in Fig. 2 (a), i.e., when only a single column of five HTS bulks are magnetized, for the first numerical model we have assumed that width of the magnetized column of bulks is much greater than its length, such that it is only necessary to consider the top view of a three columns of bulks as it is

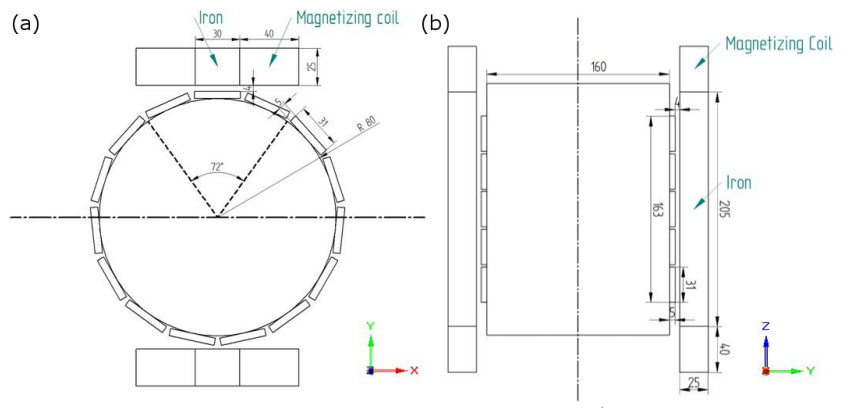

Figure 3. (Colour online) At the left, the top view of the rotor and magnetising coils is illustrated. At the right, the lateral cross-section view of the rotor and magnetising coils is shown. Dimension units are given in $\mathrm{mm}$.

shown in Fig. 3 (a). Then, in order to analyse the distribution of field along the height of the rotor, and given the fact that only the central column is strongly magnetized, a second 2D model has been created in order to consider the lateral cross section of this set of five HTS bulks (Fig. 3 (b)).

The numerical models below presented, which have been built in COMSOL Multiphysics ${ }^{\circledR}$, are based on the so-called $H$-formulation [11], and the $E-J$ power law that describes the electrical properties of the HTS bulks [12]. Each model includes two intrinsic variables. On the one hand, for the top view scheme in Fig. 3, the variables defined as $H=\left[H_{x}, H_{y}\right]^{T}$, and the input and induced currents $(J)$ flow in the $z$ direction. On the other hand, for the cross-sectional view case (Fig. 3 (b)), the variables defined as $H=\left[H_{y}, H_{z}\right]^{T}$ and the input and induced currents $(J)$ flow in the $x$ direction. In both cases, the electric field $E$ at each subdomain has the same direction as the evaluated current $J$. In the case of non-superconducting materials, the electric field is defined by the classical Ohm's law, $E=\rho \cdot J$, with $\rho=1 \times 10^{-7} \Omega \mathrm{m}$ for the iron core, $\rho=1.68 \times 10^{-8} \Omega \mathrm{m}$ for the magnetising coil (copper), and the electrical resistivity of the free-space domain (air) has been scaled to $\rho=100 \Omega \mathrm{m}$ for reducing the computing time and attaining a better numerical performance.

The actual pulsed field $B_{e x}(t)$ applied to magnetise the rotor with a rise time of $\tau=0.07 \mathrm{~s}$ and a strength of $B_{e x}^{p e a k}=1.5 \mathrm{~T}$ has been emulated by the following equation:

$$
B_{e x}(t)=B_{e x}^{\text {peak }} \frac{t}{\tau} \exp \left(1-\frac{t}{\tau}\right),
$$

Then, in order to simulate the magnetizing coil into our 2D models, it is necessary to consider the cross section dimensions of the copper winding and iron core as it is illustrated in Fig. 4. The applied current in the copper coil is implemented by a pointwise constraint such that the total current flowing through the area enclosed by the edges of this domain, is adjusted to the value of magnetic 

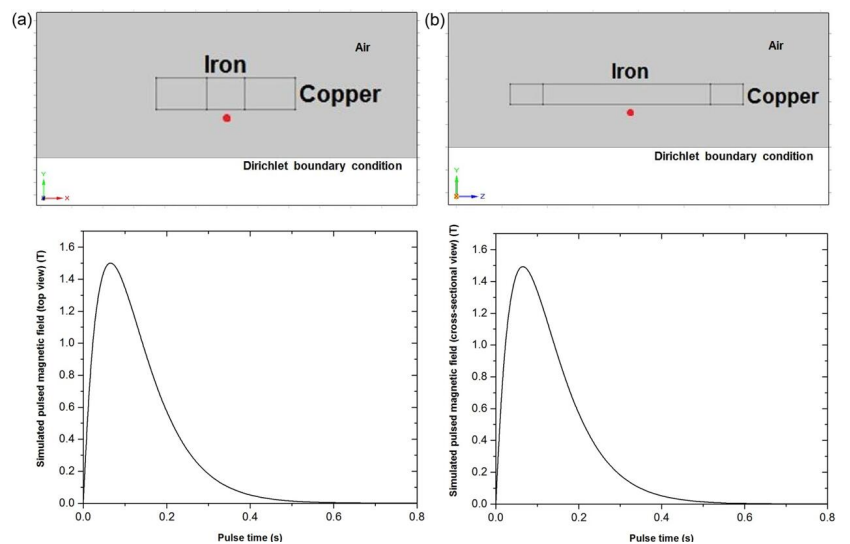

Figure 4. (Colour online) (a) At the top pane, a schematic drawing of the finite element domains for the 2D model considering the top view of magnetization coil and its iron core is shown. By symmetry only a single coil is considered and a Dirichlet boundary condition is imposed at $y=0$. At the bottom pane, the simulated pulsed magnetic field $2 \mathrm{~mm}$ away from the centre of the iron core (red dot), i.e., at the actual location of the array of hall probes in the experiment is shown. (b) Same as the left hand panes, but by considering the $2 \mathrm{D}$ model for the lateral view of the magnetization coil.

field, $B_{e x}^{\text {peak }}$, experimentally measured at the location of the hall probe. By symmetry considerations, an additional Dirichlet boundary condition $\left(H_{y}=0\right)$ is applied at the axisymmetric axis of the rotor, $80 \mathrm{~mm}$ away from the surface of the central column of HTS bulks. The other edges of the air domain are left unconstrained but with a significant larger distance from the surface of the HTS bulks. Different PDE solvers and meshing systems can be invoked within COMSOL ${ }^{\circledR}$, but for a free triangular mesh the better and faster convergence has been achieved with the Multifrontal Massively Parallel Sparse direct Solver (MUMPS), with the shape function Curl and Quadratic element order.

The highly nonlinear characteristics of the HTS bulks are described by the following $E-J$ power law,

$$
E=E_{0}\left(\frac{J}{J_{c}(B, T)}\right)^{n},
$$

where $E_{0}=100 \mu \mathrm{V} / \mathrm{m}, n=20$, and the critical current density of the HTS bulks, $J_{c}(B, T)$, depends on the intensity of the applied magnetic field temperature. This dependence has been simulated by the well stablished Kim's model [13],

$$
J_{c}(B, T)=J_{c 0}(T) \frac{B_{0}}{|B|+B_{0}},
$$

where $B$ is the local magnetic field, $J_{c 0}(T)$ is the temperature dependence critical current density for $B=0$, and $B_{0}=0.3 \mathrm{~T}$ is the average value of the magnetic field when $J_{c}$ reduces to half the magnitude of $J_{c 0}$ for our HTS
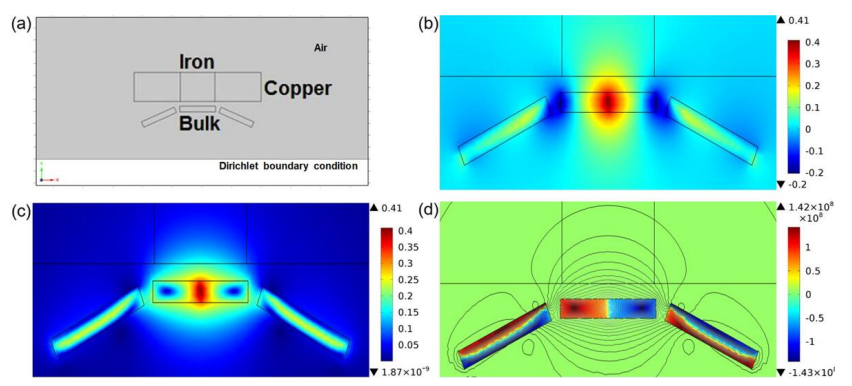

Figure 5. (a) Top view of the magnetising coil and columns of HTS bulks conforming a rotor pole as shown in Fig. 3 (a). (b) Calculated total magnetic flux density, $|B|$, after the PFM procedure. Analogously, the calculated (c) Magnetic flux density along the $y$ direction, $B_{y}$, and (d) the corresponding current density distribution are shown. Magnetic field units are in Tesla and current density is shown in $\mathrm{A} / \mathrm{m}^{2}$. (Colour online)

bulks. On the other hand, the temperature dependence of $J_{c 0}(T)$ is described by,

$$
J_{c 0}(T)=\alpha\left\{1-\left(\frac{T}{T_{c}}\right)^{2}\right\}^{\frac{3}{2}},
$$

with $T_{c}=92 \mathrm{~K}$, and $\alpha=2.1 \times 10^{9} \mathrm{~A} / \mathrm{m}^{-2}$, both in good agreement with the numerical and experimental results reported in Ref. [9]. Then, in order to calculate the instantaneous temperature of the elements, the heat transfer module of COMSOL ${ }^{\circledR}$ is coupled with our PDE model, such that the total amount of heat generated by the HTS bulks is calculated by the product $E \cdot J$, and the temperature of the outer boundaries of the system is kept at $77 \mathrm{~K}$. The physical parameters allocated into the thermal model are shown in Table 1, where the heat transfer coefficient over the surface of the superconducting bulks obeys the typical pool boiling curve for liquid nitrogen of the LN2 [14]. It is noted that although the anisotropy on superconductor materials plays a very important role when the superconductor is texturized (like in thin films of $2 \mathrm{G}$ tapes), but the $J_{c}$ in bulks is assured an average here.

Table 1. Numerical Parameters in the Heat Transfer Model

\begin{tabular}{lll}
\hline \hline Symbol & Parameter & Value \\
\hline$k$ & Thermal conductivity, HTS bulks & $14 \mathrm{~W} /(\mathrm{mK})$ \\
$\rho$ & Density, HTS bulks & $6.3 \times 10^{3} \mathrm{~kg} / \mathrm{m}^{3}$ \\
$C_{p}$ & Specific heat at constant pressure & $150 \mathrm{~J} /(\mathrm{kgK})$ \\
$h$ & Heat transfer coefficient of LN2 & Ref. [14] \\
$T_{\text {ext }}$ & Minimum temperature & $77 \mathrm{~K}$ \\
\hline \hline
\end{tabular}

After numerically matching the experimental pulsed magnetic field created by the copper coil in the two orientations illustrated in Fig. 4, the results obtained for the magnetic field and current density distributions of the HTS bulks after the PFM are shown in Figs. $5 \& 6$, 


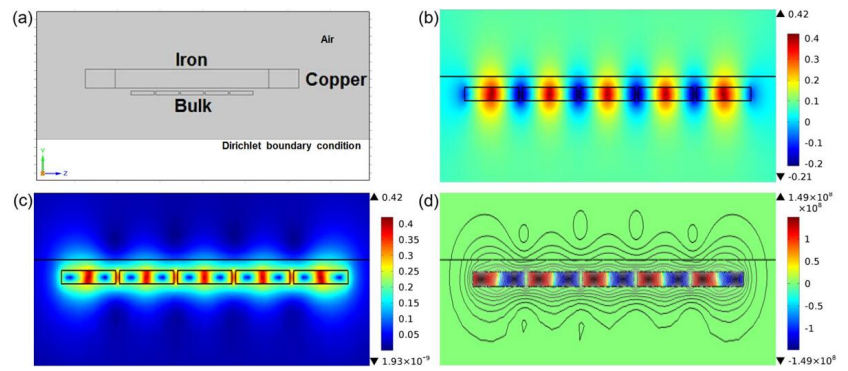

Figure 6. (Color online) Same as figure 5 but for the 2D model simulating the central column of the rotor pole along its height as it is shown in Fig. 3 (a).

respectively. It is worth mentioning that in the actual experiment, the measurements have been taken when the iron core of the magnetising coil has been removed, it after completing the PFM. Therefore, in the numerical model it is then necessary to change the relative permeability of the iron, $\mu_{r}=2 \times 10^{5}$, to $\mu_{r}=1$ (the relative permeability of air), and its electrical resistivity from $1.68 \times 10^{-8} \Omega \mathrm{m}$ to $100 \Omega \mathrm{m}$, allowing also $10 \mathrm{~min}$ for the magnetic thermal relaxation.

Thus, in order to explain why under the simplified PFM strategy, the experimental measurements have revealed that only the central column of HTS bulks at the first $\mathrm{N}$ or $\mathrm{S}$ pole are strongly magnetised along the radial direction, the current density distribution of the cross section (top-view) of the three columns of HTS bulks is displayed in Fig. 5 (d). In fact, it can be seen that the two lateral columns of HTS bulks are actually fully magnetised too, but in the azimuthal direction. In fact, it can be seen how the two lateral columns of HTS bulks are actually magnetised in the azimuthal direction, what has prevented its direct experimental observation. Moreover, because the magnetic flux produced by the magnetizing coil is nearly parallel to the magnetic field created by the distribution of currents inside of the lateral bulks, but both are flowing in opposite directions, the total magnetic field along these columns has to be significantly smaller than the observed one for the central column of bulks after the PFM procedure. In order to validate the previous statement, in Fig. 7 we show the calculated trapped magnetic flux density along the radial direction, $B_{r}$, it measured over a circumference arc $4 \mathrm{~mm}$ away from the central column of HTS bulks, i.e., at the actual location of the set of hall probes in the experiment. Starting from the left and right edges of the $\mathrm{N}$-pole, these defined in the inset of Fig. 2 at $0^{\circ}$ and $72^{\circ}$ respectively, a small peak of trapped magnetic field $(\sim 25 \mathrm{mT})$ is observed at the center of the lateral columns of HTS bulks $\left(12^{\circ}\right.$ and $\left.48^{\circ}\right)$, it in good agreement with the experimental values across the height of the pole, i.e., along the five bulks composing the entire column of HTS bulks, within a maximum deviation of about $7 \%$. Then, at the center of the pole, i.e. at the middle cross

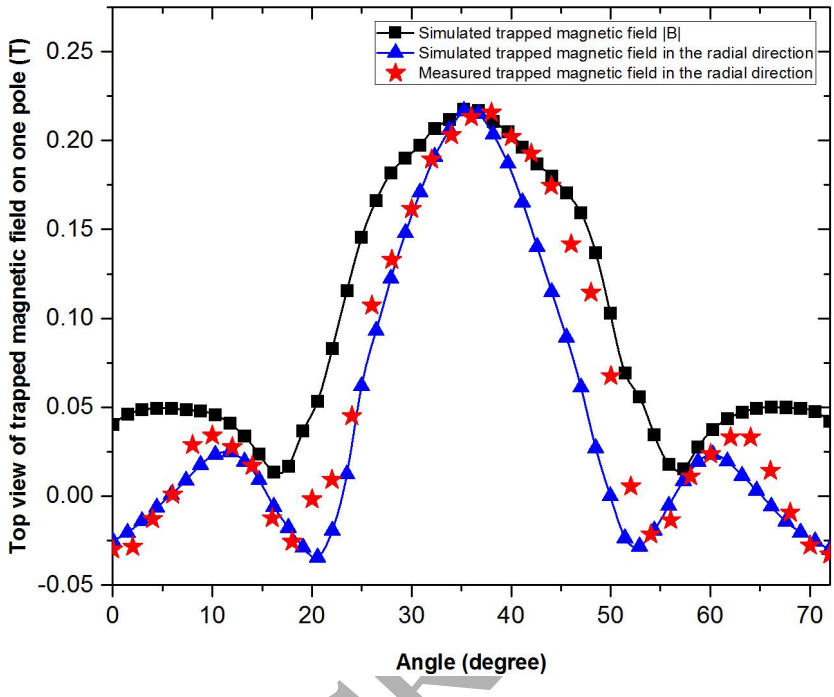

Figure 7. Comparison between the experimental (red-stars) and numerical (blue-triangles) results for the trapped magnetic field in the radial direction, $B_{r}$, it measured $4 \mathrm{~mm}$ away from for the first $\mathrm{N}$-pole displayed in Fig. 2, and accordingly with the $2 \mathrm{D}$ axisymmetric model shown in Fig. 5. The total amount of trapped magnetic field derived from the numerical model is also displayed (black-squares).

section of the central column of bulks $\left(36^{\circ}\right)$, the maximum average value for the trapped magnetic field along the radial direction $(\sim 220 \mathrm{mT})$ is reproduced with an average deviation of $2 \%$, what completes the validation of our 2D model. However, it is worth mentioning that our numerical results show slight deviations in the mean width of the peaks of trapped magnetic field that have been experimentally observed, which is a consequence of the actual differences between the diameters of the HTS bulks $(31-32 \mathrm{~mm})$, their separation distance $(0-2 \mathrm{~mm})$, as well as possible differences between their critical current densities.

Analogously, under the configuration displayed in Fig. 6, i.e., for the lateral cross section of the central column of the pole (see Fig. 3); in Fig. 8 we show the absolute value of the total trapped magnetic flux density, $|B|$, and the trapped magnetic flux density along the $y$ direction, $B_{y}$, both measured at $4 \mathrm{~mm}$ of the HTS bulks surface, and after $10 \mathrm{~min}$ of the PFM procedure to allow the magnetic thermal relaxation of the entire system. On the one hand, by comparison of the theoretical values for the maximum trapped magnetic field at the center of each bulk (243 mT, $223 \mathrm{mT}, 219 \mathrm{mT}$, $223 \mathrm{mT}$, and $243 \mathrm{mT})$, and the experimental values (232 $\mathrm{mT}, 252 \mathrm{mT}, 220 \mathrm{mT}, 241 \mathrm{mT}$, and $237 \mathrm{mT}$ ), a maximum deviation has been observed for the second and forth HTS bulks, respectively, with $\sim 11.5 \%$ and $\sim 7.5 \%$ more trapped magnetic field than the numerically expected. Nevertheless, there is an almost perfect match at the central HTS bulk with less than $0.5 \%$ deviation on the maximum intensity of trapped magnetic field. On 


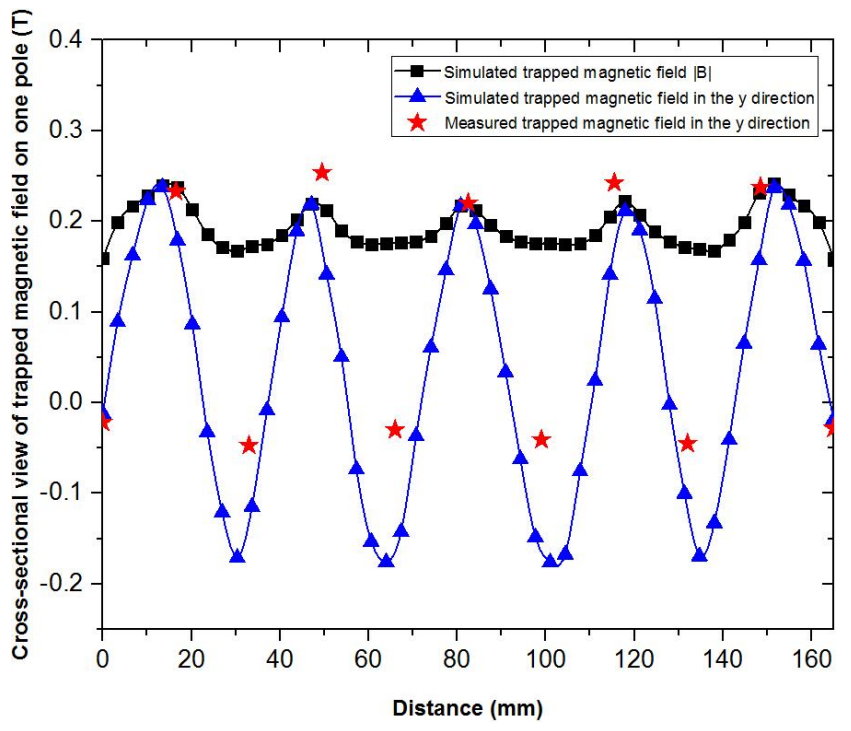

Figure 8. Comparison between the experimental (red stars) and numerical (solid line with blue triangles) results for the trapped magnetic field in the $y$ direction, $4 \mathrm{~mm}$ away from for the first N-pole displayed in Fig. 2, and accordingly with the $2 \mathrm{D}$ axisymmetric model shown in Fig. 6. The total amount of trapped magnetic field numerically calculated is also displayed (solid line with black squares).

the other hand, given the inherent size differences between the five cylindrical HTS bulks that compose the central column of the magnetic pole, and the relative position between their connecting edges and the fixed locations of the hall probes (see Fig. 1), the comparison between the $2 \mathrm{D}$ model and the experimental results has revealed how millimetre differences can difficult the accurate measurement of the minimum peaks of trapped magnetic field. Theoretically, the minimum peaks have to be observed at the connecting edges between the HTS bulks, if the HTS bulks are approached to specimens of rectangular cross section with infinite length along the $z$-axis. In the reality, these bulks are of cylindrical shape (see Fig. 1), with slightly different dimensions, physical properties, and relative positions. However, by having into consideration that the $2 \mathrm{D}$ model is actually a strong reduction of the real experimental scenario, the attained agreement between the experimental results and the numerical model is remarkable.

\section{EXTENDED PFM STRATEGY}

In the original PFM strategy (Section II), we have shown how only six out of fifteen columns of HTS bulks can be magnetized along the radial direction (see figure 2), what therefore does not make an effective use of the entire area of rotor and the other HTS bulks. Thus, in order to magnetize along the radial direction as many bulks as possible maximizing hence the effective area of

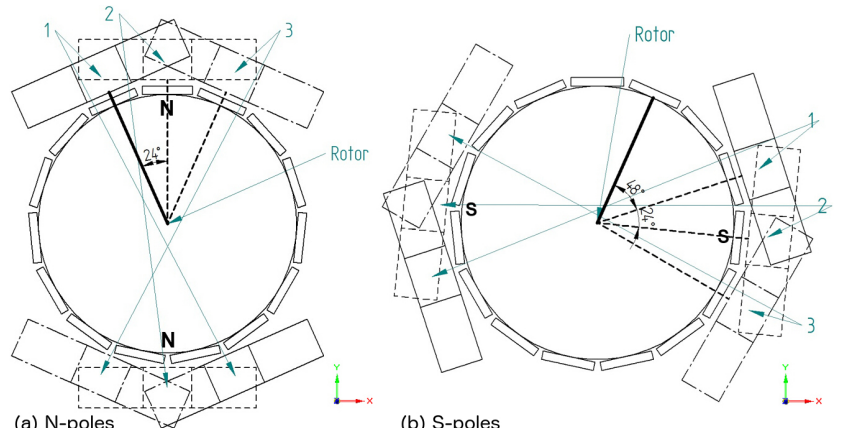

(a) $\mathrm{N}$-poles

(b) S-poles

Figure 9. The extended PFM strategy designed to produce pair of poles with larger magnetized areas. 1, 2, and 3 are the three pulse positions, starting from 1 . Two configurations are depicted: (a) produce the pair of N-poles and (b) produce the pair of S-poles.

the rotor poles, in this section an extended PFM strategy is proposed. This PFM strategy takes advantage of up to $93 \%$ of the HTS bulks, what extends the scope of the PFM procedure above discussed.

The/extended magnetization procedure is as follows: firstly, the simplified PFM strategy is applied to one of the columns of HTS bulks, i.e., by aligning the column of bulks to the magnetizing coil as it is shown in Fig. 3, and applying three pulses of magnetic field (Fig. 4), each of $B_{e x}^{\text {peak }}=1.5 \mathrm{~T}$ (see Eq. 1), with intervals of $5 \mathrm{~min}$ between each other. Then, the position of this column (labelled as 1 in Fig. 9) is rotated $24^{\circ}$ counter-clockwise, it in order to align the second column of bulks for the next PFM sequence. Thus, once two columns of bulks have been subjected to the three pulses of magnetic field each, a further $24^{\circ}$ rotation is applied such that the process is repeated for a third column of HTS bulks as shown in Fig. 9 (a). Thereby, the pair of N-poles is formed as it is can be seen in Fig. 10 (a).

Likewise, the pair of S-poles is made by the same PFM procedure described above, although the polarity of the capacitor bank charging the magnetising coil has to be inverted accordingly, and a further rotation of $48^{\circ}$ has to be implemented in order to magnetize the first column of bulks of the S-poles, as it is shown in Fig. 9 (b). Subsequently, two additional columns are magnetized, each adding a $24^{\circ}$ rotation of the rotor, what ends in the occurrence of the S-poles shown in Fig. 10 (b). The experimental measurements shown in Fig. 10 are recorded 15 min after completing the magnetization of the three columns of HTS bulks, given enough time for the magnetic relaxation of the N-poles and S-poles, respectively.

Thus, after the extended PFM strategy is applied, it is possible to state that each one of the poles is composed by at least three columns of bulks, with striking differences between the group of poles due to the uneven distribution of bulks along the surface of the rotor. For instance, in Fig. 10 (a) the first N-pole which can be defined by the first three columns of HTS bulks, i.e., between $0^{\circ}$ and 
$72^{\circ}$, showing that each one of the five bulks placed along the first of its columns (at $12^{\circ}$ ) can trap up to $190 \mathrm{mT}$, $199 \mathrm{mT}, 188 \mathrm{mT}, 196 \mathrm{mT}$, and $231 \mathrm{mT}$, at the center of each bulk respectively. Then, in the same order, the second column (at $36^{\circ}$ ) shows peaks of trapped magnetic field at $188 \mathrm{mT}, 208 \mathrm{mT}, 215 \mathrm{mT}, 187 \mathrm{mT}$, and $211 \mathrm{mT}$, respectively. Finally, the third column (at $60^{\circ}$ ) can trap up to $241 \mathrm{mT}, 230 \mathrm{mT}, 240 \mathrm{mT}, 221 \mathrm{mT}$, and $250 \mathrm{mT}$, what shows a progressive increase on the average values of trapped magnetic field per column (1st column: $196 \mathrm{mT}$, 2nd column: $208 \mathrm{mT}$, 3rd column: $196 \mathrm{mT})$, with standard deviations of $15.6 \%, 11.8 \%$, and $9.9 \%$, respectively. In fact, after these three columns have been magnetized, it is possible to observe that for the next column at the right side of the pole, each one of the bulks with centres placed at $84^{\circ}$ exhibits a small peak of trapped magnetic field along the radial direction, it of similar structure to the one shown in Fig. 7, although at the other side of the pole a much lower peak is observed due to the increase on the angle between this column and the direction of the applied magnetic field. Thus, by considering only the three columns of HTS bulks with their rotation axis aligned to the pulsed fields, the effective area of the first N-pole has been increased from at least $\sim 50.5 \mathrm{~cm}^{2}$ (1 column of HTS bulks) to $\sim 151.6 \mathrm{~cm}^{2}$.

The second N-pole lies roughly between $168^{\circ}$ and $264^{\circ}$ as shown in Fig. 10 (a). This pole shows a different pattern on the distribution of peaks when compared with the first N-pole, as consequence of the different orientation of the columns of bulks regarding the surface of the magnetizing coil. Notice the difference between the top and bottom sections of the rotor in Fig. 9 (a), where it can be seen that for the second N-pole, each one of the applied sequences of pulsed magnetic fields is performed with the center of the magnetizing coil aligned with the connecting edges between two neighbouring columns of HTS bulks. Therefore, two collateral columns are simultaneously magnetized, creating at the end an array of four columns of bulks with well defined peaks of trapped magnetic field along the radial direction. The trapped magnetic fields by these four columns are in general lower than the peaks observed for the columns of bulks pertaining to the first N-pole, as the applied magnetic field is not perpendicular to the surface of the bulks as it was the case on the magnetization of the first N-pole. Thus, the average peak values for the trapped magnetic field along the radial direction for the four columns of bulks composing the second $\mathrm{N}$-pole have been found to be $101.4 \mathrm{mT}$ $\left(180^{\circ}\right), 143.2 \mathrm{mT}\left(204^{\circ}\right), 125.6 \mathrm{mT}\left(228^{\circ}\right)$, and $91.8 \mathrm{mT}$ $\left(252^{\circ}\right)$, respectively. The two central columns of bulks have trapped more field because these columns are subjected to twice the number of magnetic pulses than the outermost columns. However, despite the area of this pole is about $50 \mathrm{~cm}^{2}$ bigger than the first N-pole, under this configuration of tilted columns of bulks for the PFM procedure, we have found that the average peak of trapped field along the columns of bulks is reduced in about a $50 \%$. This situation although far from ideal,

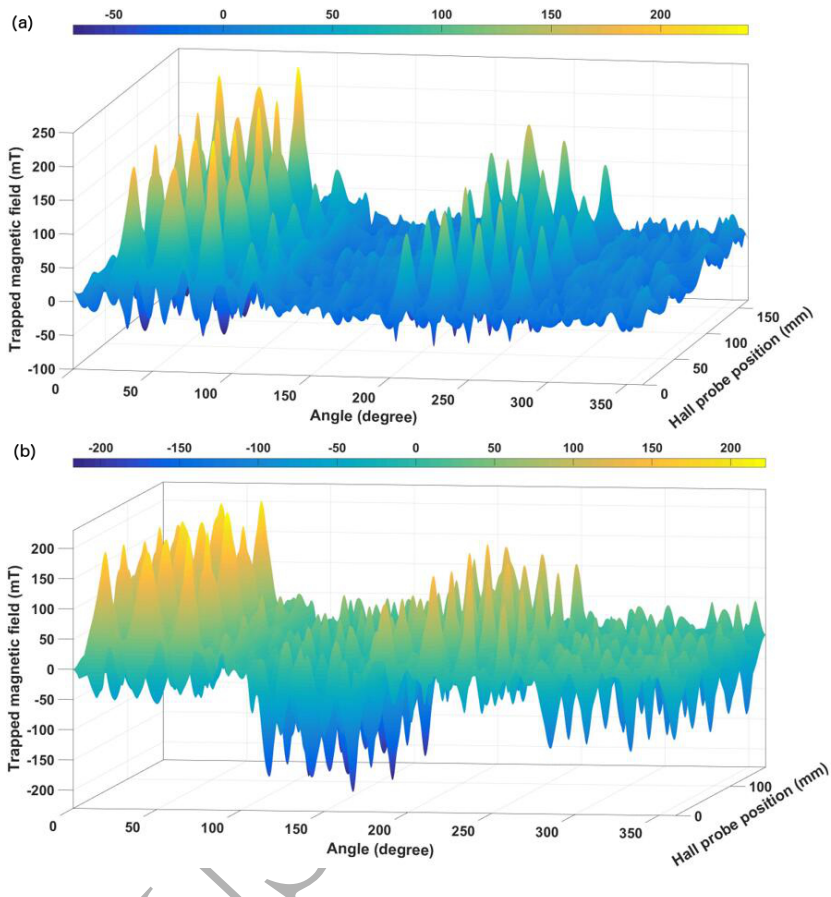

Figure $10.360^{\circ}$ field profile $\left(B_{y}\right)$ (measured $4 \mathrm{~mm}$ away from the rotor surface) of the rotor. The black box indicates the pole shape we originally designed, as seen in figure 2 . The $x$ axis is the angle position, the $y$ axis is the position along the 11 hall probes (see figure 1), and the $z$ axis is the trapped magnetic fields in the $y$ direction $\left(B_{y}\right)$. (a) After nine pulses applied to three columns as illustrated in figure 9 and (b) after subsequent nine pulses applied to three columns as illustrated in figure 9.

allows to have a clearest insight of how to design the control parameters to synchronize the rotation of the shaft with the frequency of the supply current, when there is an uneven distribution of superconducting bulks in an HTS motor.

Likewise, after the formation of the N-poles, the center of the first column of bulks for the first S-pole is identified to be at $108^{\circ}$, such that a reciprocal distribution of bulks for both S-poles is arranged, and the PFM procedure is applied in the same sequence as before (Fig. 9 (b)). Thus, given the relative orthogonality of the poles, the radial magnetization of the first $\mathrm{N}$-poles is not strongly affected by the pulse fields applied to the columns of bulks that conform the S-poles, observing a maximum reduction of the trapped magnetic field of about $12 \%$ for the third column of bulks $\left(60^{\circ}\right)$ at the first N-pole (Fig. 10). Thereby, we have successfully implemented the new PFM strategy extending the area of the superconducting magnetic poles in about a factor of three, representing a significant improvement on the utilization of superconducting bulks on the rotor designing of the HTS motor. 


\section{CONCLUSION}

In this paper we have proposed two different PFM strategies for the effective magnetization of a four-poles cylindrical rotor, it composed of an uneven distribution of superconducting bulks covering the entire active surface of the HTS rotor. Different compositions of the magnetic poles have been obtained depending on the relative orientation of the magnetizing coil and the surfaces of the columns of bulks that conform a magnetic pole. For the first PFM strategy, the experimental measurements have shown that only six out of fifteen columns of five HTS bulks each, have been magnetized along the radial direction. Thus, in order to explain these observations, two bidimensional models accounting for the electromagnetic response of the top and lateral cross sections of three columns of HTS bulks subjected to multiple pulsed magnetic fields, and after the thermal relaxation of the entire system, have been created. Both models are built in COMSOL Multiphysics ${ }^{\circledR}$, based upon the so-called HFormulation for the solution of the Maxwell equations over the superconducting domains, and the Fourier's heat transfer equation for accounting the typical pool boiling characteristics of the liquid nitrogen over their surfaces (Ref. [14]). The physical properties of the HTS bulks are described by the conventional $E-J$ power law, where the critical current density is defined by an extended version of the Kim's model, which considers the dependence with the external magnetic field and the temperature of the superconducting elements.

Our numerical results have shown an excellent agreement with the experimental observations, revealing the occurrence of a nearly orthogonal distribution of current profiles in the outer columns of a three column pole, this regarding the main direction of the magnetization in the central column which is directly subjected to the PFM. Then, in order to maximize the magnetized area of the four-pole superconducting rotor taking advantage of the whole number of HTS bulks shown in Fig. 1, an extended PFM strategy has been proposed by considering the magnetization of at least three successive columns of HTS bulks per pole. The intensity of the magnetic field and number of pulses applied to each one of the columns is the same regardless of the PFM strategy. However, in the extended PFM strategy although the area of each one of the poles can be seen increased by a factor of $200 \%-400 \%$, the maximum peaks of trapped magnetic field over the cylindrical distribution of HTS bulks can be reduced in up to a $50 \%$ of their original radial magnetization. Thus, although the effective area of the poles can be enlarged by using a significantly larger number of magnetized HTS bulks, the amount of trapped magnetic field is lower and the synchronization of the rotation of the shaft with the frequency of the supply current can be significantly challenging when there is an uneven distribution of HTS bulks over the active surface of the rotor, or when there are even slight differences between the sizes of the HTS bulks and their physical properties.
[1] Jiang Q, Majoros M, Hong Z, Campbell A and Coombs T 2006 Supercond. Sci. Technol. 191164

[2] Jiang Y, Pei R, Xian W, Hong Z and Coombs T 2008 Supercond. Sci. Technol. 21065011

[3] Huang Z, Zhang M, Wang W and Coombs T 2014 IEEE Trans. Appl. Supercond. 244602605

[4] Huang Z, et al 2013 IEEE Trans. Appl. Supercond. 23 5200204

[5] Huang Z, Ruiz H S, Zhai Y, Geng J, Shen B and Coombs T A 2016 IEEE Trans. Appl. Supercond. 265202105

[6] Matsuzaki H et al 2005 IEEE Trans. Appl. Supercond. 152222

[7] Ohtani I et at 2006 Supercond. Sci. Technol. 19 S521S524

[8] Hiroyuki Fujishiroa, Tatsuya Tateiwa, Atsushi Fujiwara,
Tetsuo Oka and Hidemi Hayashi 2006 Physica C 445-448 334

[9] Fujishiro H and Naito T 2010 Supercond. Sci. Technol. 23105021

[10] Fujishiro H, Naito T and Oyama M 2011 Supercond. Sci. Technol. 24075015

[11] Ruiz-Alonso D, Coombs T A and Campbell A M 2005 Supercond. Sci. Technol. 18 S209-S214

[12] Coombs T A, Fagnard J F and Matsuda K 2014 IEEE Trans. Appl. Supercond. 248201005

[13] Kim K B, Hempstead C F and Strnad A R 1962 Phys. Rev. Lett. 9306

[14] Haselden G G 1971 Cryogenic Fundamentals Academic Press, London-New York pp. 121 